\title{
On Generalized Pentanacci and Gaussian Generalized Pentanacci Numbers
}

\author{
Yüksel Soykan \\ Department of Mathematics, \\ Art and Science Faculty, \\ Zonguldak Bülent Ecevit University, \\ 67100, Zonguldak, Turkey \\ e-mail: yuksel_soykan@hotmail.com
}

\begin{abstract}
In this paper, we present Binet's formulas, generating functions, and the summation formulas for generalized Pentanacci numbers, and as special cases, we investigate Pentanacci and Pentanacci-Lucas numbers with their properties. Also, we define Gaussian generalized Pentanacci numbers and as special cases, we investigate Gaussian Pentanacci and Gaussian Pentanacci-Lucas numbers with their properties. Moreover, we give some identities for these numbers. Furthermore, we present matrix formulations of generalized Pentanacci numbers and Gaussian generalized Pentanacci numbers.
\end{abstract}

2010 Mathematics Subject Classification. 11B37, 11B39, 11B83.

Keywords. Pentanacci numbers, Gaussian generalized Pentanacci numbers, Gaussian Pentanacci numbers, Gaussian Pentanacci-Lucas numbers.

\section{Introduction and Preliminaries}

In this work, we investigate generalized Pentanacci numbers and give properties of Pentanacci and Pentanacci-Lucas numbers as special cases. We also define Gaussian generalized Pentanacci numbers and give properties of Gaussian Pentanacci and Gaussian Pentanacci-Lucas numbers as special cases. First, in this section, we present some background about generalized Pentanacci numbers.

There have been so many studies of the sequences of numbers in the literature which are defined recursively. Two of these type of sequences are the sequences of Pentanacci and Pentanacci-Lucas which are special case of generalized Pentanacci numbers. A generalized Pentanacci sequence $\left\{V_{n}\right\}_{n \geq 0}=\left\{V_{n}\left(V_{0}, V_{1}, V_{2}, V_{3}, V_{4}\right)\right\}_{n \geq 0}$ is defined by the fifth-order recurrence relations

$$
V_{n}=V_{n-1}+V_{n-2}+V_{n-3}+V_{n-4}+V_{n-5}
$$

with the initial values $V_{0}=c_{0}, V_{1}=c_{1}, V_{2}=c_{2}, V_{3}=c_{3}, V_{4}=c_{4}$ not all being zero. 
The sequence $\left\{V_{n}\right\}_{n \geq 0}$ can be extended to negative subscripts by defining

$$
V_{-n}=-V_{-(n-1)}-V_{-(n-2)}-V_{-(n-3)}-V_{-(n-4)}+V_{-(n-5)}
$$

for $n=1,2,3, \ldots$ Therefore, recurrence (1.1) holds for all integer $n$.

The first few generalized Pentanacci numbers with positive subscript and negative subscript are given in the following Table 1:

Table 1. A few generalized Pentanacci numbers

\begin{tabular}{ccc}
\hline$n$ & $V_{n}$ & $V_{-n}$ \\
\hline 0 & $c_{0}$ & $c_{0}$ \\
1 & $c_{1}$ & $-c_{0}-c_{1}-c_{2}-c_{3}+c_{4}$ \\
2 & $c_{2}$ & $2 c_{3}-c_{4}$ \\
3 & $c_{3}$ & $2 c_{2}-c_{3}$ \\
4 & $c_{4}$ & $2 c_{1}-c_{2}$ \\
5 & $c_{0}+c_{1}+c_{2}+c_{3}+c_{4}$ & $2 c_{0}-c_{1}$ \\
6 & $c_{0}+2 c_{1}+2 c_{2}+2 c_{3}+2 c_{4}$ & $-3 c_{0}-2 c_{1}-2 c_{2}-2 c_{3}+2 c_{4}$ \\
7 & $2 c_{0}+3 c_{1}+4 c_{2}+4 c_{3}+4 c_{4}$ & $c_{0}+c_{1}+c_{2}+5 c_{3}-3 c_{4}$ \\
8 & $4 c_{0}+6 c_{1}+7 c_{2}+8 c_{3}+8 c_{4}$ & $4 c_{2}-4 c_{3}+c_{4}$ \\
9 & $8 c_{0}+12 c_{1}+14 c_{2}+15 c_{3}+16 c_{4}$ & $4 c_{1}-4 c_{2}+c_{3}$ \\
10 & $16 c_{0}+24 c_{1}+28 c_{2}+30 c_{3}+31 c_{4}$ & $4 c_{0}-4 c_{1}+c_{2}$ \\
\hline
\end{tabular}

We consider two special cases of $V_{n}: V_{n}(0,1,1,2,4)=P_{n}$ is the sequence of Pentanacci numbers (sequence $A 001591$ in [20]) and $V_{n}(5,1,3,7,15)=Q_{n}$ is the sequence of Pentanacci-Lucas numbers $(A 074048$ in [20]). In other words, Pentanacci sequence $\left\{P_{n}\right\}_{n \geq 0}$ and Pentanacci-Lucas sequence $\left\{Q_{n}\right\}_{n \geq 0}$ are defined by the fifth-order recurrence relations

$$
P_{n}=P_{n-1}+P_{n-2}+P_{n-3}+P_{n-4}+P_{n-5}, \quad P_{0}=0, P_{1}=1, P_{2}=1, P_{3}=2, P_{4}=4
$$

and

$$
Q_{n}=Q_{n-1}+Q_{n-2}+Q_{n-3}+Q_{n-4}+Q_{n-5}, \quad Q_{0}=5, Q_{1}=1, Q_{2}=3, Q_{3}=7, Q_{4}=15
$$

respectively. Pentanacci sequence has been studied by many authors, see for example [14], [15], [19].

Next, we present the first few values of the Pentanacci and Pentanacci-Lucas numbers with positive and negative subscripts in the following Table 2 :

Table 2. A few Pentanacci and Pentanacci-Lucas Numbers

\begin{tabular}{cccccccccccccccccccccc}
$n$ & -10 & -9 & -8 & -7 & -6 & -5 & -4 & -3 & -2 & -1 & 0 & 1 & 2 & 3 & 4 & 5 & 6 & 7 & 8 & 9 & 10 \\
\hline$P_{n}$ & -3 & 2 & 0 & 0 & 0 & -1 & 1 & 0 & 0 & 0 & 0 & 1 & 1 & 2 & 4 & 8 & 16 & 31 & 61 & 120 & 236 \\
$Q_{n}$ & 19 & -1 & -1 & -1 & -7 & 9 & -1 & -1 & -1 & -1 & 5 & 1 & 3 & 7 & 15 & 31 & 57 & 113 & 223 & 439 & 863 \\
\hline
\end{tabular}


For all integers $n$, usual Pentanacci and Pentanacci-Lucas numbers can be expressed using Binet's formulas

$$
\begin{aligned}
P_{n}= & \frac{\alpha^{n+3}}{(\alpha-\beta)(\alpha-\gamma)(\alpha-\delta)(\alpha-\lambda)}+\frac{\beta^{n+3}}{(\beta-\alpha)(\beta-\gamma)(\beta-\delta)(\beta-\lambda)} \\
& +\frac{\gamma^{n+3}}{(\gamma-\alpha)(\gamma-\beta)(\gamma-\delta)(\gamma-\lambda)}+\frac{\delta^{n+3}}{(\delta-\alpha)(\delta-\beta)(\delta-\gamma)(\delta-\lambda)}+\frac{\lambda^{n+3}}{(\lambda-\alpha)(\lambda-\beta)(\lambda-\gamma)(\lambda-\delta)}
\end{aligned}
$$

(see Theorem 2.2) or

$$
P_{n}=\frac{\alpha-1}{6 \alpha-10} \alpha^{n-1}+\frac{\beta-1}{6 \beta-10} \beta^{n-1}+\frac{\gamma-1}{6 \gamma-10} \gamma^{n-1}+\frac{\delta-1}{6 \delta-10} \delta^{n-1}+\frac{\lambda-1}{6 \lambda-10} \lambda^{n-1}
$$

(see for example [5])

and

$$
Q_{n}=\alpha^{n}+\beta^{n}+\gamma^{n}+\delta^{n}+\lambda^{n}
$$

respectively, where $\alpha, \beta, \gamma, \delta$ and $\lambda$ are the roots of the equation

$$
x^{5}-x^{4}-x^{3}-x^{2}-x-1=0 .
$$

Moreover, the approximate value of $\alpha, \beta, \gamma, \delta$ and $\lambda$ are given by

$$
\begin{aligned}
& \alpha=1.9659 \\
& \beta=-0.67835+0.45854 i \\
& \gamma=-0.67835-0.45854 i \\
& \delta=0.19538+0.84885 i \\
& \lambda=0.19538-0.84885 i .
\end{aligned}
$$

In fact, there are no solutions of the characteristic equation (1.5) in terms of radicals, see [27].

Note that we have the following identities:

$$
\begin{aligned}
\alpha+\beta+\gamma+\delta+\lambda & =1, \\
\alpha \beta+\alpha \lambda+\alpha \gamma+\beta \lambda+\alpha \delta+\beta \gamma+\lambda \gamma+\beta \delta+\lambda \delta+\gamma \delta & =-1, \\
\alpha \beta \lambda+\alpha \beta \gamma+\alpha \lambda \gamma+\alpha \beta \delta+\alpha \lambda \delta+\beta \lambda \gamma+\alpha \gamma \delta+\beta \lambda \delta+\beta \gamma \delta+\lambda \gamma \delta & =1, \\
\alpha \beta \lambda \gamma+\alpha \beta \lambda \delta+\alpha \beta \gamma \delta+\alpha \lambda \gamma \delta+\beta \lambda \gamma \delta & =-1 \\
\alpha \beta \gamma \delta \lambda & =1 .
\end{aligned}
$$

\section{Properties of Generalized Pentanacci Numbers}

In this section, we present Binet's formulas, generating functions, and the summation formulas for generalized Pentanacci numbers.

First, we give the ordinary generating function $\sum_{n=0}^{\infty} V_{n} x^{n}$ of the sequence $V_{n}$. 
Lemma 2.1. Suppose that $f_{V_{n}}(x)=\sum_{n=0}^{\infty} V_{n} x^{n}$ is the ordinary generating function of the generalized Pentanacci sequence $\left\{V_{n}\right\}_{n \geq 0}$. Then $f_{V_{n}}(x)$ is given by

$$
f_{V_{n}}(x)=\frac{V_{0}+\left(V_{1}-V_{0}\right) x+\left(V_{2}-V_{1}-V_{0}\right) x^{2}+\left(V_{3}-V_{2}-V_{1}-V_{0}\right) x^{3}+\left(V_{4}-V_{3}-V_{2}-V_{1}-V_{0}\right) x^{4}}{1-x-x^{2}-x^{3}-x^{4}-x^{5}} .
$$

Proof. Using (1.1) and some calculation, we obtain

$$
\begin{aligned}
& f_{V_{n}}(x)-x f_{V_{n}}(x)-x^{2} f_{V_{n}}(x)-x^{3} f_{V_{n}}(x)-x^{4} f_{V_{n}}(x)-x^{5} f_{V_{n}}(x) \\
= & V_{0}+\left(V_{1}-V_{0}\right) x+\left(V_{2}-V_{1}-V_{0}\right) x^{2}+\left(V_{3}-V_{2}-V_{1}-V_{0}\right) x^{3}+\left(V_{4}-V_{3}-V_{2}-V_{1}-V_{0}\right) x^{4}
\end{aligned}
$$

which gives (2.1).

The previous Lemma gives the following results as particular examples: generating function of the Pentanacci sequence $P_{n}$ is

$$
f_{P_{n}}(x)=\sum_{n=0}^{\infty} P_{n} x^{n}=\frac{x}{1-x-x^{2}-x^{3}-x^{4}-x^{5}}
$$

and generating function of the Pentanacci-Lucas sequence $Q_{n}$ is

$$
f_{Q_{n}}(x)=\sum_{n=0}^{\infty} Q_{n} x^{n}=\frac{5-4 x-3 x^{2}-2 x^{3}-x^{4}}{1-x-x^{2}-x^{3}-x^{4}-x^{5}} .
$$

We next find Binet formula of Pentanacci numbers by the use of generating function for $P_{n}$.

Theorem 2.2. (Binet formula of Pentanacci numbers)

$$
\begin{aligned}
P_{n}= & \frac{\alpha^{n+3}}{(\alpha-\beta)(\alpha-\gamma)(\alpha-\delta)(\alpha-\lambda)}+\frac{\beta^{n+3}}{(\beta-\alpha)(\beta-\gamma)(\beta-\delta)(\beta-\lambda)} \\
& +\frac{\gamma^{n+3}}{(\gamma-\alpha)(\gamma-\beta)(\gamma-\delta)(\gamma-\lambda)}+\frac{\delta^{n+3}}{(\delta-\alpha)(\delta-\beta)(\delta-\gamma)(\delta-\lambda)} \\
& +\frac{\lambda^{n+3}}{(\lambda-\alpha)(\lambda-\beta)(\lambda-\gamma)(\lambda-\delta)} .
\end{aligned}
$$

Proof. Let

$$
h(x)=1-x-x^{2}-x^{3}-x^{4}-x^{5} .
$$

Then for some $\alpha, \beta, \gamma, \delta$ and $\lambda$, we write

$$
h(x)=(1-\alpha x)(1-\beta x)(1-\gamma x)(1-\delta x)(1-\lambda x)
$$

i.e.,

$$
1-x-x^{2}-x^{3}-x^{4}-x^{5}=(1-\alpha x)(1-\beta x)(1-\gamma x)(1-\delta x)(1-\lambda x)
$$

Hence $\frac{1}{\alpha}, \frac{1}{\beta}, \frac{1}{\gamma}, \frac{1}{\delta}$ ve $\frac{1}{\lambda}$ are the roots of $h(x)$. This gives $\alpha, \beta, \gamma, \delta$ and $\lambda$ as the roots of

$$
h\left(\frac{1}{x}\right)=1-\frac{1}{x}-\frac{1}{x^{2}}-\frac{1}{x^{3}}-\frac{1}{x^{4}}-\frac{1}{x^{5}}=0 .
$$


This implies $x^{5}-x^{4}-x^{3}-x^{2}-x-1=0$. Now, by (2.2) and (2.5), it follows that

$$
f_{P_{n}}(x)=\sum_{n=0}^{\infty} P_{n} x^{n}=\frac{x}{(1-\alpha x)(1-\beta x)(1-\gamma x)(1-\delta x)(1-\lambda x)} .
$$

Then we write

$$
\frac{x}{(1-\alpha x)(1-\beta x)(1-\gamma x)(1-\delta x)(1-\lambda x)}=\frac{A}{(1-\alpha x)}+\frac{B}{(1-\beta x)}+\frac{C}{(1-\gamma x)}+\frac{D}{(1-\delta x)}+\frac{E}{(1-\lambda x)}
$$

So

$$
\begin{aligned}
x= & A(1-\beta x)(1-\gamma x)(1-\delta x)(1-\lambda x)+B(1-\alpha x)(1-\gamma x)(1-\delta x)(1-\lambda x) \\
& +C(1-\alpha x)(1-\beta x)(1-\delta x)(1-\lambda x)+D(1-\alpha x)(1-\beta x)(1-\gamma x)(1-\lambda x) \\
& +E(1-\alpha x)(1-\beta x)(1-\gamma x)(1-\delta x) .
\end{aligned}
$$

If we consider $x=\frac{1}{\alpha}$, we get $\frac{1}{\alpha}=A\left(1-\frac{\beta}{\alpha}\right)\left(1-\frac{\gamma}{\alpha}\right)\left(1-\frac{\delta}{\alpha}\right)\left(1-\frac{\lambda}{\alpha}\right)$. This gives $A=\frac{\alpha^{3}}{(\alpha-\beta)(\alpha-\gamma)(\alpha-\delta)(\alpha-\lambda)}$.

Similarly, we obtain

$$
\begin{aligned}
& B=\frac{\beta^{3}}{(\beta-\alpha)(\beta-\gamma)(\beta-\delta)(\beta-\lambda)}, C=\frac{\gamma^{3}}{(\gamma-\alpha)(\gamma-\beta)(\gamma-\delta)(\gamma-\lambda)}, \\
& D=\frac{\delta^{3}}{(\delta-\alpha)(\delta-\beta)(\delta-\gamma)(\delta-\lambda)}, E=\frac{\lambda^{3}}{(\lambda-\alpha)(\lambda-\beta)(\lambda-\gamma)(\lambda-\delta)} .
\end{aligned}
$$

Thus (2.6) can be written as

$$
f_{P_{n}}(x)=A(1-\alpha x)^{-1}+B(1-\beta x)^{-1}+C(1-\gamma x)^{-1}+D(1-\delta x)^{-1}+E(1-\lambda x)^{-1} .
$$

This gives

$$
\begin{aligned}
f_{P_{n}}(x) & =A \sum_{n=0}^{\infty} \alpha^{n} x^{n}+B \sum_{n=0}^{\infty} \beta^{n} x^{n}+C \sum_{n=0}^{\infty} \gamma^{n} x^{n}+D \sum_{n=0}^{\infty} \delta^{n} x^{n}+E \sum_{n=0}^{\infty} \lambda^{n} x^{n} \\
& =\sum_{n=0}^{\infty}\left(A \alpha^{n}+B \beta^{n}+C \gamma^{n}+D \delta^{n}+E \lambda^{n}\right) x^{n} .
\end{aligned}
$$

Using the values of $A, B, C, D$ and $E$ we get

$$
\begin{aligned}
f_{P_{n}}(x)= & \sum_{n=0}^{\infty} P_{n} x^{n}=\sum_{n=0}^{\infty}\left(\frac{\alpha^{3}}{(\alpha-\beta)(\alpha-\gamma)(\alpha-\delta)(\alpha-\lambda)}\right. \\
& +\frac{\beta^{n+3}}{(\beta-\alpha)(\beta-\gamma)(\beta-\delta)(\beta-\lambda)}+\frac{\gamma^{3}}{(\gamma-\alpha)(\gamma-\beta)(\gamma-\delta)(\gamma-\lambda)} \\
& \left.+\frac{\delta^{3}}{(\delta-\alpha)(\delta-\beta)(\delta-\gamma)(\delta-\lambda)}+\frac{\lambda^{3}}{(\lambda-\alpha)(\lambda-\beta)(\lambda-\gamma)(\lambda-\delta)}\right) x^{n}
\end{aligned}
$$

Therefore, comparing coefficients on both sides of the above equality, we get (2.4).

Next, we give an identity related with generalized Pentanacci numbers and Pentanacci numbers.

Theorem 2.3. For $n \geq 0$ and $m \geq 0$, the following identity holds:

$V_{m+n}=P_{m-4} V_{n}+\left(P_{m-4}+P_{m-5}\right) V_{n+1}+\left(P_{m-4}+P_{m-5}+P_{m-6}\right) V_{n+2}+\left(P_{m-4}+P_{m-5}+P_{m-6}+P_{m-7}\right) V_{n+3}+P_{m-3} V_{n+4}$ 
Proof. We prove the identity by induction on $m$. If $m=0$ then

$$
V_{n}=P_{-4} V_{n}+\left(P_{-4}+P_{-5}\right) V_{n+1}+\left(P_{-4}+P_{-5}+P_{-6}\right) V_{n+2}+\left(P_{-4}+P_{-5}+P_{-6}+P_{-7}\right) V_{n+3}+P_{-3} V_{n+4}
$$

which is true because $P_{-3}=0, P_{-4}=1, P_{-5}=-1, P_{-6}=0, P_{-7}=0$. Assume that the equaliy holds for all $m \leq k$. For $m=k+1$, we have

$$
\begin{aligned}
V_{(k+1)+n}= & V_{n+k}+V_{n+k-1}+V_{n+k-2}+V_{n+k-3}+V_{n+k-4} \\
= & \left(P_{k-4} V_{n}+\left(P_{k-4}+P_{k-5}\right) V_{n+1}+\left(P_{k-4}+P_{k-5}+P_{k-6}\right) V_{n+2}\right. \\
& \left.+\left(P_{k-4}+P_{k-5}+P_{k-6}+P_{k-7}\right) V_{n+3}+P_{k-3} V_{n+4}\right) \\
& +\left(P_{k-5} V_{n}+\left(P_{k-5}+P_{k-6}\right) V_{n+1}+\left(P_{k-5}+P_{k-6}+P_{k-7}\right) V_{n+2}\right. \\
& \left.+\left(P_{k-5}+P_{k-6}+P_{k-7}+P_{k-8}\right) V_{n+3}+P_{k-4} V_{n+4}\right) \\
& +\left(P_{k-6} V_{n}+\left(P_{k-6}+P_{k-7}\right) V_{n+1}+\left(P_{k-6}+P_{k-7}+P_{k-8}\right) V_{n+2}\right. \\
& \left.+\left(P_{k-6}+P_{k-7}+P_{k-8}+P_{k-9}\right) V_{n+3}+P_{k-5} V_{n+4}\right) \\
& +\left(P_{k-7} V_{n}+\left(P_{k-7}+P_{k-8}\right) V_{n+1}+\left(P_{k-7}+P_{k-8}+P_{k-9}\right) V_{n+2}\right. \\
& \left.+\left(P_{k-7}+P_{k-8}+P_{k-9}+P_{k-10}\right) V_{n+3}+P_{k-6} V_{n+4}\right) \\
& +\left(P_{k-8} V_{n}+\left(P_{k-8}+P_{k-9}\right) V_{n+1}+\left(P_{k-8}+P_{k-9}+P_{k-10}\right) V_{n+2}\right. \\
& \left.+\left(P_{k-8}+P_{k-9}+P_{k-10}+P_{k-11}\right) V_{n+3}+P_{k-7} V_{n+4}\right) \\
= & \left(P_{k-3} V_{n}+\left(P_{k-3}+P_{k-4}\right) V_{n+1}+\left(P_{k-3}+P_{k-4}+P_{k-5}\right) V_{n+2}\right. \\
& \left.+\left(P_{k-3}+P_{k-4}+P_{k-5}+P_{k-6}\right) V_{n+3}+P_{k-2} V_{n+4}\right) \\
= & P_{(k+1)-4} V_{n}+\left(P_{(k+1)-4}+P_{(k+1)-5}\right) V_{n+1}+\left(P_{(k+1)-4}+P_{(k+1)-5}+P_{(k+1)-6}\right) V_{n+2} \\
& +\left(P_{(k+1)-4}+P_{(k+1)-5}+P_{(k+1)-6}+P_{(k+1)-7}\right) V_{n+3}+P_{(k+1)-3} V_{n+4}
\end{aligned}
$$

By induction on $m$, this proves (2.7).

The previous Theorem gives the following results as particular examples: For $n \geq 0$ and $m \geq 0$, we have (taking $V_{n}=P_{n}$ )

$P_{m+n}=P_{m-4} P_{n}+\left(P_{m-4}+P_{m-5}\right) P_{n+1}+\left(P_{m-4}+P_{m-5}+P_{m-6}\right) P_{n+2}+\left(P_{m-4}+P_{m-5}+P_{m-6}+P_{m-7}\right) P_{n+3}+P_{m-3} P_{n+4}$ and (taking $V_{n}=Q_{n}$ )

$Q_{m+n}=P_{m-4} Q_{n}+\left(P_{m-4}+P_{m-5}\right) Q_{n+1}+\left(P_{m-4}+P_{m-5}+P_{m-6}\right) Q_{n+2}+\left(P_{m-4}+P_{m-5}+P_{m-6}+P_{m-7}\right) Q_{n+3}+P_{m-3} Q_{n+4}$.

Next we present the Binet's formula of the generalized Pentanacci sequence.

Lemma 2.4. The Binet's formula of the generalized Pentanacci sequence $\left\{V_{n}\right\}$ is given as $V_{n}=P_{n-4} V_{0}+\left(P_{n-4}+P_{n-5}\right) V_{1}+\left(P_{n-4}+P_{n-5}+P_{n-6}\right) V_{2}+\left(P_{n-4}+P_{n-5}+P_{n-6}+P_{n-7}\right) V_{3}+P_{n-3} V_{4}$. 
Proof. Take $n=0$ and then replace $n$ with $m$ in Theorem 2.3 .

For another proof of the Lemma 2.4, see [19]. This Lemma is also a special case of a work on the $n$th $k$-generalized Fibonacci number (which is also called $k$-step Fibonacci number) in [2, Theorem 2.2].

Corollary 2.5. The Binet's formula of the generalized Pentanacci sequence $\left\{V_{n}\right\}$ is given as

$$
V_{n}=A_{1} \alpha^{n-8}+A_{2} \beta^{n-8}+A_{3} \gamma^{n-8}+A_{4} \delta^{n-8}+A_{5} \lambda^{n-8}
$$

where

$$
\begin{aligned}
& A_{1}=\frac{\alpha-1}{6 \alpha-10}\left(V_{4} \alpha^{4}+\left(V_{0}+V_{1}+V_{2}+V_{3}\right) \alpha^{3}+\left(V_{1}+V_{2}+V_{3}\right) \alpha^{2}+\left(V_{2}+V_{3}\right) \alpha+V_{3}\right), \\
& A_{2}=\frac{\beta-1}{6 \beta-10}\left(V_{4} \beta^{4}+\left(V_{0}+V_{1}+V_{2}+V_{3}\right) \beta^{3}+\left(V_{1}+V_{2}+V_{3}\right) \beta^{2}+\left(V_{2}+V_{3}\right) \beta+V_{3}\right), \\
& A_{3}=\frac{\gamma-1}{6 \gamma-10}\left(V_{4} \gamma^{4}+\left(V_{0}+V_{1}+V_{2}+V_{3}\right) \gamma^{3}+\left(V_{1}+V_{2}+V_{3}\right) \gamma^{2}+\left(V_{2}+V_{3}\right) \gamma+V_{3}\right), \\
& A_{4}=\frac{\delta-1}{6 \delta-10}\left(V_{4} \delta^{4}+\left(V_{0}+V_{1}+V_{2}+V_{3}\right) \delta^{3}+\left(V_{1}+V_{2}+V_{3}\right) \delta^{2}+\left(V_{2}+V_{3}\right) \delta+V_{3}\right), \\
& A_{5}=\frac{\lambda-1}{6 \lambda-10}\left(V_{4} \lambda^{4}+\left(V_{0}+V_{1}+V_{2}+V_{3}\right) \lambda^{3}+\left(V_{1}+V_{2}+V_{3}\right) \lambda^{2}+\left(V_{2}+V_{3}\right) \lambda+V_{3}\right) .
\end{aligned}
$$

Proof. The proof follows from Lemma 2.4 and (1.4).

In fact, Corollary 2.5 is a special case of a result in [2, Remark 2.3].

The following Theorem present some summation formulas of generalized Pentanacci numbers.

TheOREM 2.6. For $n \geq 1$ we have the following summing formulas:

(a): (Sum of the generalized Pentanacci numbers)

$$
\sum_{k=1}^{n} V_{k}=\frac{1}{4}\left(V_{n+4}-V_{n+2}-2 V_{n+1}+V_{n}-V_{4}+V_{2}+2 V_{1}-V_{0}\right)
$$

(b): $\sum_{k=1}^{n} V_{2 k+1}=\frac{1}{8}\left(3 V_{2 n+2}+4 V_{2 n+1}+V_{2 n}+2 V_{2 n-1}-V_{2 n-2}-3 V_{4}+4 V_{3}-V_{2}-2 V_{1}+V_{0}\right)$

(c): $\sum_{k=1}^{n} V_{2 k}=\frac{1}{8}\left(-V_{2 n+2}+4 V_{2 n+1}+5 V_{2 n}+2 V_{2 n-1}+3 V_{2 n-2}+V_{4}-4 V_{3}+3 V_{2}-2 V_{1}-3 V_{0}\right)$.

Proof.

(a): Using the recurrence relation

$$
V_{n}=V_{n-1}+V_{n-2}+V_{n-3}+V_{n-4}+V_{n-5}
$$

i.e.

$$
V_{n-5}=V_{n}-V_{n-1}-V_{n-2}-V_{n-3}-V_{n-4}
$$


we obtain

$$
\begin{aligned}
V_{0}= & V_{5}-V_{4}-V_{3}-V_{2}-V_{1} \\
V_{1}= & V_{6}-V_{5}-V_{4}-V_{3}-V_{2} \\
V_{2}= & V_{7}-V_{6}-V_{5}-V_{4}-V_{3} \\
V_{3}= & V_{8}-V_{7}-V_{6}-V_{5}-V_{4} \\
V_{4}= & V_{9}-V_{8}-V_{7}-V_{6}-V_{5} \\
& \vdots \\
V_{n-5}= & V_{n}-V_{n-1}-V_{n-2}-V_{n-3}-V_{n-4} \\
V_{n-4}= & V_{n+1}-V_{n}-V_{n-1}-V_{n-2}-V_{n-3} \\
V_{n-3}= & V_{n+2}-V_{n+1}-V_{n}-V_{n-1}-V_{n-2} \\
V_{n-2}= & V_{n+3}-V_{n+2}-V_{n+1}-V_{n}-V_{n-1} \\
V_{n-1}= & V_{n+4}-V_{n+3}-V_{n+2}-V_{n+1}-V_{n} \\
V_{n}= & V_{n+5}-V_{n+4}-V_{n+3}-V_{n+2}-V_{n+1} .
\end{aligned}
$$

If we add the equations by side by, we get

$$
\begin{aligned}
V_{0}+\sum_{k=1}^{n} V_{k}= & \left(-V_{4}-V_{3}-V_{2}-V_{1}+\sum_{k=1}^{n} V_{k}\right)+\left(V_{3}+V_{2}+V_{1}-\sum_{k=1}^{n} V_{k}\right) \\
& +\left(V_{2}+V_{1}-\sum_{k=1}^{n} V_{k}\right)+\left(V_{1}-\sum_{k=1}^{n} V_{k}\right)+\left(-\sum_{k=1}^{n} V_{k}\right) \\
& -3 V_{n+1}-2 V_{n+2}-V_{n+3}+V_{n+5} \\
= & -3 V_{n+1}-2 V_{n+2}-V_{n+3}+V_{n+5}+\left(-V_{4}+V_{2}+2 V_{1}\right)-3 \sum_{k=1}^{n} V_{k}
\end{aligned}
$$

and then

$$
4 \sum_{k=1}^{n} V_{k}=V_{n+5}-V_{n+3}-2 V_{n+2}-3 V_{n+1}-V_{4}+V_{2}+2 V_{1}-V_{0}
$$

It follows that

$$
\begin{aligned}
\sum_{k=1}^{n} V_{k} & =\frac{1}{4}\left(V_{n+5}-V_{n+3}-2 V_{n+2}-3 V_{n+1}-V_{4}+V_{2}+2 V_{1}-V_{0}\right) \\
& =\frac{1}{4}\left(\left(V_{n+4}+V_{n+3}+V_{n+2}+V_{n+1}+V_{n}\right)-V_{n+3}-2 V_{n+2}-3 V_{n+1}-V_{4}+V_{2}+2 V_{1}-V_{0}\right) \\
& =\frac{1}{4}\left(V_{n+4}-V_{n+2}-2 V_{n+1}+V_{n}-V_{4}+V_{2}+2 V_{1}-V_{0}\right) .
\end{aligned}
$$


(b): When we use (3.1), we obtain the following equalities:

$$
\begin{aligned}
V_{k}= & V_{k-1}+V_{k-2}+V_{k-3}+V_{k-4}+V_{k-5} \\
V_{4}= & V_{3}+V_{2}+V_{1}+V_{0}+V_{-1} \\
V_{6}= & V_{5}+V_{4}+V_{3}+V_{2}+V_{1} \\
V_{8}= & V_{7}+V_{6}+V_{5}+V_{4}+V_{3} \\
V_{10}= & V_{9}+V_{8}+V_{7}+V_{6}+V_{5} \\
& \vdots \\
V_{2 n}= & V_{2 n-1}+V_{2 n-2}+V_{2 n-3}+V_{2 n-4}+V_{2 n-5} \\
V_{2 n+2}= & V_{2 n+1}+V_{2 n}+V_{2 n-1}+V_{2 n-2}+V_{2 n-3} .
\end{aligned}
$$

If we rearrange the above equalities, we obtain

$$
\begin{aligned}
V_{3}= & V_{4}-V_{2}-V_{1}-V_{0}-V_{-1} \\
V_{5}= & V_{6}-V_{4}-V_{3}-V_{2}-V_{1} \\
V_{7}= & V_{8}-V_{6}-V_{5}-V_{4}-V_{3} \\
V_{9}= & V_{10}-V_{8}-V_{7}-V_{6}-V_{5} \\
V_{11}= & V_{12}-V_{10}-V_{9}-V_{8}-V_{7} \\
V_{13}= & V_{14}-V_{12}-V_{11}-V_{10}-V_{9} \\
V_{15}= & V_{16}-V_{14}-V_{13}-V_{12}-V_{11} \\
& \vdots \\
V_{2 n-1}= & V_{2 n}-V_{2 n-2}-V_{2 n-3}-V_{2 n-4}-V_{2 n-5} \\
V_{2 n+1}= & V_{2 n+2}-V_{2 n}-V_{2 n-1}-V_{2 n-2}-V_{2 n-3} .
\end{aligned}
$$

Now, if we add the above equations by side by, we get (as a I. Method)

$$
\begin{aligned}
\sum_{k=1}^{n} V_{2 k+1} & =-V_{-1}-V_{0}-V_{2}+\left(-V_{1}-V_{3}-V_{5}-V_{7}-\ldots-V_{2 n-1}\right)-\left(\sum_{k=1}^{2 n-2} V_{k}\right)+V_{2 n+2} \\
& =-V_{-1}-V_{0}-V_{2}+\left(V_{2 n+1}-V_{1}-\sum_{k=1}^{n} V_{2 k+1}\right)-\left(\sum_{k=1}^{2 n-2} V_{k}\right)+V_{2 n+2}
\end{aligned}
$$

and then, using (a), we obtain

$$
\sum_{k=1}^{n} V_{2 k+1}=\frac{1}{8}\left(3 V_{2 n+2}+4 V_{2 n+1}+V_{2 n}+2 V_{2 n-1}-V_{2 n-2}-3 V_{4}+4 V_{3}-V_{2}-2 V_{1}+V_{0}\right)
$$


Note that, as an alternative method (II. Method), the following equality can be used:

$$
\begin{aligned}
\sum_{k=1}^{n} V_{2 k+1}= & \left(-V_{2}+V_{2 n+2}+\sum_{k=1}^{n} V_{2 k}\right)+\left(-\sum_{k=1}^{n} V_{2 k}\right)+\left(-V_{1}+V_{2 n+1}-\sum_{k=1}^{n} V_{2 k+1}\right) \\
& +\left(-V_{0}+V_{2 n}-\sum_{k=1}^{n} V_{2 k}\right)+\left(-V_{-1}-V_{1}+V_{2 n-1}+V_{2 n+1}-\sum_{k=1}^{n} V_{2 k+1}\right) .
\end{aligned}
$$

(c): (c) follows from (a), (b) and the equality

$$
\sum_{k=1}^{n} V_{2 k}=\sum_{k=1}^{2 n+1} V_{k}-\sum_{k=1}^{n} V_{2 k+1}-V_{1} .
$$

This completes the proof.

All the listed identities in Theorem 2.6 may be proved by induction, but that method of proof gives no clue about their discovery.

As special cases of the above Theorem, we have the following two Corollaries. First one present some summing formulas of Pentanacci numbers.

Corollary 2.7. For $n \geq 1$ we have the following formulas:

(a): (Sum of the Pentanacci numbers)

$$
\sum_{k=1}^{n} P_{k}=\frac{1}{4}\left(P_{n+4}-P_{n+2}-2 P_{n+1}+P_{n}-1\right)
$$

(b): $\sum_{k=1}^{n} P_{2 k+1}=\frac{1}{8}\left(3 P_{2 n+2}+4 P_{2 n+1}+P_{2 n}+2 P_{2 n-1}-P_{2 n-2}-7\right)$

(c): $\sum_{k=1}^{n} P_{2 k}=\frac{1}{8}\left(-P_{2 n+2}+4 P_{2 n+1}+5 P_{2 n}+2 P_{2 n-1}+3 P_{2 n-2}-3\right)$.

Second Corollary gives some summing formulas of Pentanacci-Lucas numbers.

Corollary 2.8. For $n \geq 1$ we have the following formulas:

(a): (Sum of the Pentanacci-Lucas numbers)

$$
\sum_{k=1}^{n} Q_{k}=\frac{1}{4}\left(Q_{n+4}-Q_{n+2}-2 Q_{n+1}+Q_{n}-15\right)
$$

(b): $\sum_{k=1}^{n} Q_{2 k+1}=\frac{1}{8}\left(3 Q_{2 n+2}+4 Q_{2 n+1}+Q_{2 n}+2 Q_{2 n-1}-Q_{2 n-2}-17\right)$

(c): $\sum_{k=1}^{n} Q_{2 k}=\frac{1}{8}\left(-Q_{2 n+2}+4 Q_{2 n+1}+5 Q_{2 n}+2 Q_{2 n-1}+3 Q_{2 n-2}-21\right)$.

Sometimes, we need to start summing from zero, such as when dealing with Pentanacci quaternions, sedenions, etc. We can state Theorem 2.6 in the following form.

Theorem 2.9. For $n \geq 0$, we have the following formulas:

(a): $\sum_{k=0}^{n} V_{k}=\frac{1}{4}\left(V_{n+4}-V_{n+2}-2 V_{n+1}+V_{n}-V_{4}+V_{2}+2 V_{1}+3 V_{0}\right)$,

(b): $\sum_{k=0}^{n} V_{2 k+1}=\frac{1}{8}\left(3 V_{2 n+2}+4 V_{2 n+1}+V_{2 n}+2 V_{2 n-1}-V_{2 n-2}-3 V_{4}+4 V_{3}-V_{2}+6 V_{1}+V_{0}\right)$

(c): $\sum_{k=0}^{n} V_{2 k}=\frac{1}{8}\left(-V_{2 n+2}+4 V_{2 n+1}+5 V_{2 n}+2 V_{2 n-1}+3 V_{2 n-2}+V_{4}-4 V_{3}+3 V_{2}-2 V_{1}+5 V_{0}\right)$. 
As special cases of above Theorem, we have the following two Corollaries. First one present some summation formulas of Pentanacci numbers.

Corollary 2.10. For $n \geq 0$, we have the following formulas:

(a): $\sum_{k=0}^{n} P_{k}=\frac{1}{4}\left(P_{n+4}-P_{n+2}-2 P_{n+1}+P_{n}-1\right)$

(b): $\sum_{k=0}^{n} P_{2 k+1}=\frac{1}{8}\left(3 P_{2 n+2}+4 P_{2 n+1}+P_{2 n}+2 P_{2 n-1}-P_{2 n-2}+1\right)$

(c): $\sum_{k=0}^{n} P_{2 k}=\frac{1}{8}\left(-P_{2 n+2}+4 P_{2 n+1}+5 P_{2 n}+2 P_{2 n-1}+3 P_{2 n-2}-3\right)$.

Next Corollary gives some summation formulas of Pentanacci-Lucas numbers.

Corollary 2.11. For $n \geq 0$, we have the following formulas:

(a): $\sum_{k=0}^{n} Q_{k}=\frac{1}{4}\left(Q_{n+4}-Q_{n+2}-2 Q_{n+1}+Q_{n}+5\right)$

(b): $\sum_{k=0}^{n} Q_{2 k+1}=\frac{1}{8}\left(3 Q_{2 n+2}+4 Q_{2 n+1}+Q_{2 n}+2 Q_{2 n-1}-Q_{2 n-2}-9\right)$

(c): $\sum_{k=0}^{n} Q_{2 k}=\frac{1}{8}\left(-Q_{2 n+2}+4 Q_{2 n+1}+5 Q_{2 n}+2 Q_{2 n-1}+3 Q_{2 n-2}+19\right)$.

\section{Gaussian Generalized Pentanacci Numbers}

In this section, we introduce Gaussian generalized Pentanacci numbers and present Binet's formulas, generating functions, and the summation formulas for Gaussian generalized Pentanacci numbers.

First we recall Gaussian integers. A Gaussian integer $z$ is a complex number whose real and imaginary parts are both integers, i.e., $z=a+i b, a, b \in \mathbb{Z}$. These numbers is denoted by $\mathbb{Z}[i]$. For more information about this kind of integers, see the work of Fraleigh [6].

If we use together sequences of integers defined recursively and Gaussian type integers, we obtain a new sequences of complex numbers such as Gaussian Fibonacci, Gaussian Lucas, Gaussian Pell, Gaussian PellLucas and Gaussian Jacobsthal numbers; Gaussian Padovan and Gaussian Pell-Padovan numbers; Gaussian Tribonacci numbers.

In 1963, Horadam [11] introduced the concept of complex Fibonacci number called as the Gaussian Fibonacci number. Pethe [17] defined the complex Tribonacci numbers at Gaussian integers, see also [7]. There are other several studies dedicated to these sequences of Gaussian numbers such as the works in [1], [3], [4], [8], [9], [10], [12], [13], [16], [21], [22], [24], [25], [26], among others.

Gaussian generalized Pentanacci numbers $\left\{G V_{n}\right\}_{n \geq 0}=\left\{G V_{n}\left(G V_{0}, G V_{1}, G V_{2}, G V_{3}, G V_{4}\right)\right\}_{n \geq 0}$ are defined by

$$
G V_{n}=G V_{n-1}+G V_{n-2}+G V_{n-3}+G V_{n-4}+G V_{n-5},
$$

with the initial conditions

$$
\begin{aligned}
& G V_{0}=c_{0}+\left(-c_{0}-c_{1}-c_{2}-c_{3}+c_{4}\right) i, G V_{1}=c_{1}+c_{0} i, G V_{2}=c_{2}+c_{1} i \\
& G V_{3}=c_{3}+c_{2} i, G V_{4}=c_{4}+c_{3} i
\end{aligned}
$$


not all being zero. The sequences $\left\{G V_{n}\right\}_{n \geq 0}$ can be extended to negative subscripts by defining

$$
G V_{-n}=-G V_{-(n-1)}-G V_{-(n-2)}-G V_{-(n-3)}-G V_{-(n-4)}+G V_{-(n-5)}
$$

for $n=1,2,3, \ldots$ Therefore, recurrence (3.1) hold for all integer $n$. Note that for $n \geq 0$

$$
G V_{n}=V_{n}+i V_{n-1}
$$

and

$$
G V_{-n}=V_{-n}+i V_{-n-1}
$$

The first few generalized Gaussian Pentanacci numbers with positive subscript and negative subscript are given in the following Table 3 and Table 4 :

Table 3. A few Gaussian generalized Pentanacci numbers with positive subscript

\begin{tabular}{lc}
$n$ & $G V_{n}$ \\
\hline 0 & $c_{0}+\left(-c_{0}-c_{1}-c_{2}-c_{3}+c_{4}\right) i$ \\
1 & $c_{1}+c_{0} i$ \\
2 & $c_{2}+c_{1} i$ \\
3 & $c_{3}+c_{2} i$ \\
4 & $c_{4}+c_{3} i$ \\
5 & $\left(c_{0}+c_{1}+c_{2}+c_{3}+c_{4}\right)+c_{4} i$ \\
6 & $\left(c_{0}+2 c_{1}+2 c_{2}+2 c_{3}+2 c_{4}\right)+\left(c_{0}+c_{1}+c_{2}+c_{3}+c_{4}\right) i$ \\
7 & $\left(2 c_{0}+3 c_{1}+4 c_{2}+4 c_{3}+4 c_{4}\right)+\left(c_{0}+2 c_{1}+2 c_{2}+2 c_{3}+2 c_{4}\right) i$ \\
8 & $\left(4 c_{0}+6 c_{1}+7 c_{2}+8 c_{3}+8 c_{4}\right)+\left(2 c_{0}+3 c_{1}+4 c_{2}+4 c_{3}+4 c_{4}\right) i$ \\
9 & $\left(8 c_{0}+12 c_{1}+14 c_{2}+15 c_{3}+16 c_{4}\right)+\left(4 c_{0}+6 c_{1}+7 c_{2}+8 c_{3}+8 c_{4}\right) i$ \\
10 & $\left(16 c_{0}+24 c_{1}+28 c_{2}+30 c_{3}+31 c_{4}\right)+\left(8 c_{0}+12 c_{1}+14 c_{2}+15 c_{3}+16 c_{4}\right) i$ \\
\hline
\end{tabular}

Table 4. A few Gaussian generalized Pentanacci numbers with negative subscript 


\begin{tabular}{lc}
\hline$n$ & $G V_{-n}$ \\
\hline 0 & $c_{0}+\left(-c_{0}-c_{1}-c_{2}-c_{3}+c_{4}\right) i$ \\
1 & $\left(-c_{0}-c_{1}-c_{2}-c_{3}+c_{4}\right)+\left(2 c_{3}-c_{4}\right) i$ \\
2 & $\left(2 c_{3}-c_{4}\right)+\left(2 c_{2}-c_{3}\right) i$ \\
3 & $\left(2 c_{2}-c_{3}\right)+\left(2 c_{1}-c_{2}\right) i$ \\
4 & $\left(2 c_{1}-c_{2}\right)+\left(2 c_{0}-c_{1}\right) i$ \\
5 & $\left(2 c_{0}-c_{1}\right)+\left(-3 c_{0}-2 c_{1}-2 c_{2}-2 c_{3}+2 c_{4}\right) i$ \\
6 & $\left(-3 c_{0}-2 c_{1}-2 c_{2}-2 c_{3}+2 c_{4}\right)+\left(c_{0}+c_{1}+c_{2}+5 c_{3}-3 c_{4}\right) i$ \\
7 & $\left(c_{0}+c_{1}+c_{2}+5 c_{3}-3 c_{4}\right)+\left(4 c_{2}-4 c_{3}+c_{4}\right) i$ \\
8 & $\left(4 c_{2}-4 c_{3}+c_{4}\right)+\left(4 c_{1}-4 c_{2}+c_{3}\right) i$ \\
9 & $\left(4 c_{1}-4 c_{2}+c_{3}\right)+\left(4 c_{0}-4 c_{1}+c_{2}\right) i$ \\
10 & $\left(4 c_{0}-4 c_{1}+c_{2}\right)+\left(4 c_{4}-3 c_{1}-4 c_{2}-4 c_{3}-8 c_{0}\right) i$ \\
\hline
\end{tabular}

We consider two special cases of $G V_{n}: G V_{n}(0,1,1+i, 2+i, 4+2 i)=G P_{n}$ is the sequence of Gaussian Pentanacci numbers and $G V_{n}(5-i, 1+5 i, 3+i, 7+3 i, 15+7 i)=G Q_{n}$ is the sequence of Gaussian PentanacciLucas numbers. We formally define them as follows:

Gaussian Pentanacci numbers are defined by

$$
G P_{n}=G P_{n-1}+G P_{n-2}+G P_{n-3}+G P_{n-4}+G P_{n-5}
$$

with the initial conditions

$$
G P_{0}=0, G P_{1}=1, G P_{2}=1+i, G P_{3}=2+i, G P_{4}=4+2 i
$$

and Gaussian Pentanacci-Lucas numbers are defined by

$$
G Q_{n}=G Q_{n-1}+G Q_{n-2}+G Q_{n-3}+G Q_{n-4}+G Q_{n-5}
$$

with the initial conditions

$$
G Q_{0}=5-i, G Q_{1}=1+5 i, G Q_{2}=3+i, G Q_{3}=7+3 i, G Q_{4}=15+7 i
$$

Note that for $n \geq 0$

$$
G P_{n}=M_{n}+i M_{n-1}, G Q_{n}=R_{n}+i R_{n-1}
$$

and

$$
G P_{-n}=M_{-n}+i M_{-n-1}, G Q_{-n}=R_{-n}+i R_{-n-1} .
$$

Next, we present the first few values of the Gaussian Pentanacci and Pentanacci-Lucas numbers with positive and negative subscripts in the following Table 4:

Table 4. A few Gaussian Pentanacci and Pentanacci-Lucas Numbers 


\begin{tabular}{ccccccccccc}
\hline$n$ & 0 & 1 & 2 & 3 & 4 & 5 & 6 & 7 & 8 & 9 \\
\hline$G P_{n}$ & 0 & 1 & $1+i$ & $2+i$ & $4+2 i$ & $8+4 i$ & $16+8 i$ & $31+16 i$ & $61+31 i$ & $120+61 i$ \\
$G P_{-n}$ & 0 & 0 & 0 & $i$ & $1-i$ & -1 & 0 & 0 & $2 i$ & $2-3 i$ \\
$G Q_{n}$ & $5-i$ & $1+5 i$ & $3+i$ & $7+3 i$ & $15+7 i$ & $31+15 i$ & $57+31 i$ & $113+57 i$ & $223+113 i$ & $439+223 i$ \\
$G Q_{-n}$ & $5-i$ & $-1-i$ & $-1-i$ & $-1-i$ & $-1+9 i$ & $9-7 i$ & $-7-i$ & $-1-i$ & $-1-i$ & $-1+19 i$ \\
\hline
\end{tabular}

The following Theorem presents the generating function $f_{G V_{n}}(x)=\sum_{n=0}^{\infty} G V x^{n}$ of Gaussian generalized Pentanacci numbers $G V_{n}$.

Theorem 3.1. The generating function of Gaussian generalized Pentanacci numbers is given as

$$
f_{G V_{n}}(x)=\frac{\begin{array}{c}
G V_{0}+\left(G V_{1}-G V_{0}\right) x+\left(G V_{2}-G V_{1}-G V_{0}\right) x^{2}+\left(G V_{3}-G V_{2}-G V_{1}-G V_{0}\right) x^{3} \\
+\left(G V_{4}-G V_{3}-G V_{2}-G V_{1}-G V_{0}\right) x^{4}
\end{array}}{1-x-x^{2}-x^{3}-x^{4}-x^{5}} .
$$

Proof. Using (3.1) and some calculation, we obtain

$$
\begin{aligned}
& f_{G V_{n}}(x)-x f_{G V_{n}}(x)-x^{2} f_{G V_{n}}(x)-x^{3} f_{G V_{n}}(x)-x^{4} f_{G V_{n}}(x)-x^{5} f_{G V_{n}}(x) \\
= & G V_{0}+\left(G V_{1}-G V_{0}\right) x+\left(G V_{2}-G V_{1}-G V_{0}\right) x^{2}+\left(G V_{3}-G V_{2}-G V_{1}-G V_{0}\right) x^{3} \\
& +\left(G V_{4}-G V_{3}-G V_{2}-G V_{1}-G V_{0}\right) x^{4}
\end{aligned}
$$

which gives (3.5).

The previous Theorem gives the following results as particular examples: the generating function of Gaussian Pentanacci numbers is

$$
f_{G P_{n}}(x)=\frac{x+i x^{2}}{1-x-x^{2}-x^{3}-x^{4}-x^{5}}
$$

and the generating function of Gaussian Pentanacci-Lucas numbers is

$$
f_{G Q_{n}}(x)=\frac{5-i-(4-6 i) x-(3+3 i) x^{2}-(2+2 i) x^{3}-(1+i) x^{4}}{1-x-x^{2}-x^{3}-x^{4}-x^{5}} .
$$

We now present the Binet formula for the Gaussian generalized Pentanacci numbers.

Theorem 3.2. The Binet formula for the Gaussian generalized Pentanacci numbers is

$$
\begin{aligned}
G V_{n}= & \left(A_{1} \alpha^{n-8}+A_{2} \beta^{n-8}+A_{3} \gamma^{n-8}+A_{4} \delta^{n-8}+A_{5} \lambda^{n-8}\right) \\
& +i\left(A_{1} \alpha^{n-9}+A_{2} \beta^{n-9}+A_{3} \gamma^{n-9}+A_{4} \delta^{n-9}+A_{5} \lambda^{n-9}\right)
\end{aligned}
$$

where $A_{1}, A_{2}, A_{3}, A_{4}$ and $A_{5}$ are as in Corollary 2.5.

Proof. The proof follows from Corollary 2.5 and $G V_{n}=V_{n}+i V_{n-1}$. 
The previous Theorem gives the following results as particular examples: the Binet formula for the Gaussian Pentanacci numbers is

$$
\begin{aligned}
G P_{n}= & \left(\frac{\alpha^{n+3}}{(\alpha-\beta)(\alpha-\gamma)(\alpha-\delta)(\alpha-\lambda)}+\frac{\beta^{n+3}}{(\beta-\alpha)(\beta-\gamma)(\beta-\delta)(\beta-\lambda)}+\frac{\gamma^{n+3}}{(\gamma-\alpha)(\gamma-\beta)(\gamma-\delta)(\gamma-\lambda)}\right. \\
& \left.+\frac{\lambda^{n+3}}{(\delta-\alpha)(\delta-\beta)(\delta-\gamma)(\delta-\lambda)}+\frac{\alpha^{n+2}}{(\lambda-\alpha)(\lambda-\beta)(\lambda-\gamma)(\lambda-\delta)}\right) \\
& +i\left(\frac{\beta^{n+2}}{(\alpha-\beta)(\alpha-\gamma)(\alpha-\delta)(\alpha-\lambda)}+\frac{\gamma^{n+2}}{(\beta-\alpha)(\beta-\gamma)(\beta-\delta)(\beta-\lambda)}+\frac{\lambda^{n+2}}{(\gamma-\alpha)(\gamma-\beta)(\gamma-\delta)(\gamma-\lambda)}\right. \\
& +\frac{\delta^{n+2}}{(\delta-\alpha)(\delta-\beta)(\delta-\gamma)(\delta-\lambda)}+\frac{\left.\lambda^{n+\alpha)(\lambda-\beta)(\lambda-\gamma)(\lambda-\delta)}\right)}{(\lambda-\alpha)}
\end{aligned}
$$

or

$$
\begin{aligned}
G P_{n}= & \left(\frac{\alpha-1}{6 \alpha-10} \alpha^{n-1}+\frac{\beta-1}{6 \beta-10} \beta^{n-1}+\frac{\gamma-1}{6 \gamma-10} \gamma^{n-1}+\frac{\delta-1}{6 \delta-10} \delta^{n-1}+\frac{\lambda-1}{6 \lambda-10} \lambda^{n-1}\right) \\
& +i\left(\frac{\alpha-1}{6 \alpha-10} \alpha^{n-2}+\frac{\beta-1}{6 \beta-10} \beta^{n-2}+\frac{\gamma-1}{6 \gamma-10} \gamma^{n-2}+\frac{\delta-1}{6 \delta-10} \delta^{n-2}+\frac{\lambda-1}{6 \lambda-10} \lambda^{n-2}\right)
\end{aligned}
$$

and the Binet formula for the Gaussian Pentanacci-Lucas numbers is

$$
G Q_{n}=\left(\alpha^{n}+\beta^{n}+\gamma^{n}+\delta^{n}+\lambda^{n}\right)+i\left(\alpha^{n-1}+\beta^{n-1}+\gamma^{n-1}+\delta^{n-1}+\lambda^{n-1}\right) .
$$

The following Theorem present some summation formulas of Gaussian generalized Pentanacci numbers.

THEOREM 3.3. For $n \geq 1$ we have the following formulas:

(a): (Sum of the Gaussian generalized Pentanacci numbers)

$$
\sum_{k=1}^{n} G V_{k}=\frac{1}{4}\left(G V_{n+4}-G V_{n+2}-2 G V_{n+1}+G V_{n}-G V_{4}+G V_{2}+2 G V_{1}-G V_{0}\right)
$$

(b): $\sum_{k=1}^{n} G V_{2 k+1}=\frac{1}{8}\left(3 G V_{2 n+2}+4 G V_{2 n+1}+G V_{2 n}+2 G V_{2 n-1}-G V_{2 n-2}-3 G V_{4}+4 G V_{3}-G V_{2}-\right.$ $\left.2 G V_{1}+G V_{0}\right)$

(c): $\sum_{k=1}^{n} G V_{2 k}=\frac{1}{8}\left(-G V_{2 n+2}+4 G V_{2 n+1}+5 G V_{2 n}+2 G V_{2 n-1}+3 G V_{2 n-2}+G V_{4}-4 G V_{3}+3 G V_{2}-\right.$ $\left.2 G V_{1}-3 G V_{0}\right)$.

Proof. (a), (b) and (c) can be proved exactly as in the proof of Theorem 2.6.

As special cases of the above Theorem, we have the following two Corollaries. First one present summation formulas of Gaussian Pentanacci numbers.

COROLLARY 3.4. For $n \geq 1$ we have the following formulas:

(a): (Sum of the Gaussian Pentanacci numbers)

$$
\sum_{k=1}^{n} G P_{k}=\frac{1}{4}\left(G P_{n+4}-G P_{n+2}-2 G P_{n+1}+G P_{n}-1-i\right)
$$

(b): $\sum_{k=1}^{n} G P_{2 k+1}=\frac{1}{8}\left(3 G P_{2 n+2}+4 G P_{2 n+1}+G P_{2 n}+2 G P_{2 n-1}-G P_{2 n-2}-7-3 i\right)$

(c): $\sum_{k=1}^{n} G P_{2 k}=\frac{1}{8}\left(-G P_{2 n+2}+4 G P_{2 n+1}+5 G P_{2 n}+2 G P_{2 n-1}+3 G P_{2 n-2}-3+i\right)$. 
Second Corollary gives summation formulas of Gaussian Pentanacci-Lucas numbers.

COROLlary 3.5. For $n \geq 1$ we have the following formulas:

(a): (Sum of the Gaussian Pentanacci-Lucas numbers)

$$
\sum_{k=1}^{n} G Q_{k}=\frac{1}{4}\left(G Q_{n+4}-G Q_{n+2}-2 G Q_{n+1}+G Q_{n}-15+5 i\right)
$$

(b): $\sum_{k=1}^{n} G Q_{2 k+1}=\frac{1}{8}\left(3 G Q_{2 n+2}+4 G Q_{2 n+1}+G Q_{2 n}+2 G Q_{2 n-1}-G Q_{2 n-2}-17-21 i\right)$

(c): $\sum_{k=1}^{n} G Q_{2 k}=\frac{1}{8}\left(-G Q_{2 n+2}+4 G Q_{2 n+1}+5 G Q_{2 n}+2 G Q_{2 n-1}+3 G Q_{2 n-2}-21-9 i\right)$.

\section{Basic Relations and Simson Formulas}

In this section, we obtain some identities of Pentanacci numbers and Pentanacci-Lucas numbers and some identities of Gaussian Pentanacci numbers and Gaussian Pentanacci-Lucas numbers. Moreover, we present Simson formulas of these numbers.

First, we can give a few basic relations between $\left\{P_{n}\right\}$ and $\left\{Q_{n}\right\}$.

THEOREM 4.1. The following equalities are true:

$$
\begin{aligned}
Q_{n} & =-P_{n+3}+7 P_{n+1}-2 P_{n}-P_{n-1}, \\
Q_{n} & =-P_{n+2}+6 P_{n+1}-3 P_{n}-2 P_{n-1}-P_{n-2}, \\
Q_{n} & =3 P_{n+4}-7 P_{n+2}-9 P_{n}-8 P_{n-1}-4 P_{n-2}, \\
Q_{2 n+1} & =287 P_{n+2}-286 P_{n+1}-281 P_{n}-264 P_{n-1}-213 P_{n-2},
\end{aligned}
$$

and

$$
\begin{aligned}
& 1198 P_{n}=27 Q_{n+3}+18 Q_{n+2}+3 Q_{n+1}-22 Q_{n}+136 Q_{n-1} \\
& 1198 P_{n}=45 Q_{n+3}-15 Q_{n+1}-40 Q_{n}+118 Q_{n-1}-18 Q_{n-2}
\end{aligned}
$$

Proof. Note that the last six identities hold for all integers $n$. For example, to show (4.1), writing

$$
Q_{n}=a P_{n+3}+b P_{n+2}+c P_{n+1}+d P_{n}+e P_{n-1}
$$

and solving the system of equations

$$
\begin{aligned}
& Q_{0}=a P_{3}+b P_{2}+c P_{1}+d P_{0}+e P_{-1} \\
& Q_{1}=a P_{4}+b P_{3}+c P_{2}+d P_{1}+e P_{0} \\
& Q_{2}=a P_{5}+b P_{4}+c P_{3}+d P_{2}+e P_{1} \\
& Q_{3}=a P_{6}+b P_{5}+c P_{4}+d P_{3}+e P_{2} \\
& Q_{4}=a P_{7}+b P_{6}+c P_{5}+d P_{4}+e P_{3}
\end{aligned}
$$

we find that $a=-1, b=0, c=7, d=-2, e=-1$. The other equalities can be proved similarly. 
We present a few basic relations between $\left\{G P_{n}\right\}$ and $\left\{G Q_{n}\right\}$.

THeOREM 4.2. The following equalities are true:

$$
\begin{aligned}
G Q_{n} & =-G P_{n+3}+7 G P_{n+1}-2 G P_{n}-G P_{n-1}, \\
G Q_{n} & =-G P_{n+2}+6 G P_{n+1}-3 G P_{n}-2 G P_{n-1}-G P_{n-2}, \\
G Q_{n} & =3 G P_{n+4}-7 G P_{n+2}-9 G P_{n}-8 G P_{n-1}-4 G P_{n-2}, \\
G Q_{2 n+1} & =287 G P_{n+2}-286 G P_{n+1}-281 G P_{n}-264 G P_{n-1}-213 G P_{n-2},
\end{aligned}
$$

and

$$
\begin{aligned}
& 1198 G P_{n}=27 G Q_{n+3}+18 G Q_{n+2}+3 G Q_{n+1}-22 G Q_{n}+136 G Q_{n-1} \\
& 1198 G P_{n}=45 G Q_{n+3}-15 G Q_{n+1}-40 G Q_{n}+118 G Q_{n-1}-18 G Q_{n-2}
\end{aligned}
$$

Proof. Note that the last six identities hold for all integers $n$. For example, to show (4.7), writing

$$
G Q_{n}=a G P_{n+3}+b G P_{n+2}+c G P_{n+1}+d G P_{n}+e G P_{n-1}
$$

and solving the system of equations

$$
\begin{aligned}
G Q_{0} & =a G P_{3}+b G P_{2}+c G P_{1}+d G P_{0}+e G P_{-1} \\
G Q_{1} & =a G P_{4}+b G P_{3}+c G P_{2}+d G P_{1}+e G P_{0} \\
G Q_{2} & =a G P_{5}+b G P_{4}+c G P_{3}+d G P_{2}+e G P_{1} \\
G Q_{3} & =a G P_{6}+b G P_{5}+c G P_{4}+d G P_{3}+e G P_{2} \\
G Q_{4} & =a G P_{7}+b G P_{6}+c G P_{5}+d G P_{4}+e G P_{3}
\end{aligned}
$$

we find that $a=-1, b=0, c=7, d=-2, e=-1$. Or using the relations $G P_{n}=P_{n}+i P_{n-1}, G Q_{n}=$ $Q_{n}+i Q_{n-1}$ and identity $Q_{n}=-P_{n+3}+7 P_{n+1}-2 P_{n}-P_{n-1}$ (see Theorem 4.1) we obtain the identity (4.7). In fact, note that

$$
\begin{aligned}
G Q_{n} & =Q_{n}+i Q_{n-1} \\
& =\left(-P_{n+3}+7 P_{n+1}-2 P_{n}-P_{n-1}\right)+i\left(-P_{n+2}+7 P_{n}-2 P_{n-1}-P_{n-2}\right) \\
& =-\left(P_{n+3}+i P_{n+2}\right)+7\left(P_{n+1}+i P_{n}\right)-2\left(P_{n}+i P_{n-1}\right)-\left(P_{n-1}+i P_{n-2}\right) \\
& =-G P_{n+3}+7 G P_{n+1}-2 G P_{n}-G P_{n-1} .
\end{aligned}
$$

The other equalities can be proved similarly.

We can also give a few basic relations between $\left\{G Q_{n}\right\}$ and $\left\{P_{n}\right\}$.

$$
\begin{aligned}
G Q_{n} & =(5-i) P_{n+1}-(4-6 i) P_{n}-(3+3 i) P_{n-1}-(2+2 i) P_{n-2}-(1+i) P_{n-3} \\
G Q_{n+4} & =(15+7 i) P_{n+1}+(16+8 i) P_{n}+(11+9 i) P_{n-1}+(10+4 i) P_{n-2}+(7+3 i) P_{n-3}
\end{aligned}
$$


We present an identity related with Gaussian generalized Pentanacci numbers and Pentanacci numbers.

TheOrem 4.3. For $n \geq 0$ and $m \geq 0$, the following identity holds:

$$
\begin{aligned}
G V_{m+n}= & P_{m-4} G V_{n}+\left(P_{m-4}+P_{m-5}\right) G V_{n+1}+\left(P_{m-4}+P_{m-5}+P_{m-6}\right) G V_{n+2} \\
& +\left(P_{m-4}+P_{m-5}+P_{m-6}+P_{m-7}\right) G V_{n+3}+P_{m-3} G V_{n+4}
\end{aligned}
$$

Proof. The identity (4.15) can be proved by induction on $m$ as in Theorem 2.3.

The previous Theorem gives the following results as particular examples: For $n \geq 0$ and $m \geq 0$, we have (taking $G V_{n}=G P_{n}$ )

$$
\begin{aligned}
G P_{m+n}= & P_{m-4} G P_{n}+\left(P_{m-4}+P_{m-5}\right) G P_{n+1}+\left(P_{m-4}+P_{m-5}+P_{m-6}\right) G P_{n+2} \\
& +\left(P_{m-4}+P_{m-5}+P_{m-6}+P_{m-7}\right) G P_{n+3}+P_{m-3} G P_{n+4}
\end{aligned}
$$

and (taking $G V_{n}=G Q_{n}$ )

$$
\begin{aligned}
G Q_{m+n}= & P_{m-4} G Q_{n}+\left(P_{m-4}+P_{m-5}\right) G Q_{n+1}+\left(P_{m-4}+P_{m-5}+P_{m-6}\right) G Q_{n+2} \\
& +\left(P_{m-4}+P_{m-5}+P_{m-6}+P_{m-7}\right) G Q_{n+3}+P_{m-3} G Q_{n+4} .
\end{aligned}
$$

One of the oldest and best known identities for the Fibonacci sequence $\left\{F_{n}\right\}$ is

$$
F_{n+1} F_{n-1}-F_{n}^{2}=(-1)^{n}
$$

which was derived first by R. Simson in 1753 [18]. This can be written in the form

$$
\left|\begin{array}{cc}
F_{n+1} & F_{n} \\
F_{n} & F_{n-1}
\end{array}\right|=(-1)^{n}
$$

and called as Simson or Cassini formula (Identity). The following Theorem gives generalization of this result to generalized Pentanacci numbers.

Theorem 4.4. (Simson's formula of generalized Pentanacci numbers) For all integers n, we have

$$
\left|\begin{array}{ccccc}
V_{n+4} & V_{n+3} & V_{n+2} & V_{n+1} & V_{n} \\
V_{n+3} & V_{n+2} & V_{n+1} & V_{n} & V_{n-1} \\
V_{n+2} & V_{n+1} & V_{n} & V_{n-1} & V_{n-2} \\
V_{n+1} & V_{n} & V_{n-1} & V_{n-2} & V_{n-3} \\
V_{n} & V_{n-1} & V_{n-2} & V_{n-3} & V_{n-4}
\end{array}\right|=\left|\begin{array}{ccccc}
V_{4} & V_{3} & V_{2} & V_{1} & V_{0} \\
V_{3} & V_{2} & V_{1} & V_{0} & V_{-1} \\
V_{2} & V_{1} & V_{0} & V_{-1} & V_{-2} \\
V_{1} & V_{0} & V_{-1} & V_{-2} & V_{-3} \\
V_{0} & V_{-1} & V_{-2} & V_{-3} & V_{-4}
\end{array}\right| .
$$

Proof. (4.17) is given in Soykan [23].

COROLlary 4.5. For all integers n, we have 
(a): (Simson's formula of Pentanacci numbers)

$$
\left|\begin{array}{ccccc}
P_{n+4} & P_{n+3} & P_{n+2} & P_{n+1} & P_{n} \\
P_{n+3} & P_{n+2} & P_{n+1} & P_{n} & P_{n-1} \\
P_{n+2} & P_{n+1} & P_{n} & P_{n-1} & P_{n-2} \\
P_{n+1} & P_{n} & P_{n-1} & P_{n-2} & P_{n-3} \\
P_{n} & P_{n-1} & P_{n-2} & P_{n-3} & P_{n-4}
\end{array}\right|=1
$$

(b): (Simpson's formula of Pentanacci-Lucas numbers)

$$
\left|\begin{array}{ccccc}
Q_{n+4} & Q_{n+3} & Q_{n+2} & Q_{n+1} & Q_{n} \\
Q_{n+3} & Q_{n+2} & Q_{n+1} & Q_{n} & Q_{n-1} \\
Q_{n+2} & Q_{n+1} & Q_{n} & Q_{n-1} & Q_{n-2} \\
Q_{n+1} & Q_{n} & Q_{n-1} & Q_{n-2} & Q_{n-3} \\
Q_{n} & Q_{n-1} & Q_{n-2} & Q_{n-3} & Q_{n-4}
\end{array}\right|=9584
$$

Note that Simson's formula of Gaussian Pentanacci numbers is

$$
\left|\begin{array}{ccccc}
G P_{n+4} & G P_{n+3} & G P_{n+2} & G P_{n+1} & G P_{n} \\
G P_{n+3} & G P_{n+2} & G P_{n+1} & G P_{n} & G P_{n-1} \\
G P_{n+2} & G P_{n+1} & G P_{n} & G P_{n-1} & G P_{n-2} \\
G P_{n+1} & G P_{n} & G P_{n-1} & G P_{n-2} & G P_{n-3} \\
G P_{n} & G P_{n-1} & G P_{n-2} & G P_{n-3} & G P_{n-4}
\end{array}\right|=1+i
$$

and Simson's formula of Gaussian Pentanacci-Lucas numbers is

$$
\left|\begin{array}{ccccc}
G Q_{n+4} & G Q_{n+3} & G Q_{n+2} & G Q_{n+1} & G Q_{n} \\
G Q_{n+3} & G Q_{n+2} & G Q_{n+1} & G Q_{n} & G Q_{n-1} \\
G Q_{n+2} & G Q_{n+1} & G Q_{n} & G Q_{n-1} & G Q_{n-2} \\
G Q_{n+1} & G Q_{n} & G Q_{n-1} & G Q_{n-2} & G Q_{n-3} \\
G Q_{n} & G Q_{n-1} & G Q_{n-2} & G Q_{n-3} & G Q_{n-4}
\end{array}\right|=9584+9584 i
$$

\section{Matrix Formulations of $V_{n}$ and $G V_{n}$}

In this section, we present some matrix formulation of generalized Pentanacci numbers and Gaussian generalized Pentanacci numbers.

We define the square matrix $A$ of order 5 as:

$$
A=\left(\begin{array}{lllll}
1 & 1 & 1 & 1 & 1 \\
1 & 0 & 0 & 0 & 0 \\
0 & 1 & 0 & 0 & 0 \\
0 & 0 & 1 & 0 & 0 \\
0 & 0 & 0 & 1 & 0
\end{array}\right)
$$


such that $\operatorname{det} A=1$. Induction proof may be used to establish

$$
A^{n}=\left(\begin{array}{ccccc}
P_{n+1} & P_{n}+P_{n-1}+P_{n-2}+P_{n-3} & P_{n}+P_{n-1}+P_{n-2} & P_{n}+P_{n-1} & P_{n} \\
P_{n} & P_{n-1}+P_{n-2}+P_{n-3}+P_{n-4} & P_{n-1}+P_{n-2}+P_{n-3} & P_{n-1}+P_{n-2} & P_{n-1} \\
P_{n-1} & P_{n-2}+P_{n-3}+P_{n-4}+P_{n-5} & P_{n-2}+P_{n-3}+P_{n-4} & P_{n-2}+P_{n-3} & P_{n-2} \\
P_{n-2} & P_{n-3}+P_{n-4}+P_{n-5}+P_{n-6} & P_{n-3}+P_{n-4}+P_{n-5} & P_{n-3}+P_{n-4} & P_{n-3} \\
P_{n-3} & P_{n-4}+P_{n-5}+P_{n-6}+P_{n-7} & P_{n-4}+P_{n-5}+P_{n-6} & P_{n-4}+P_{n-5} & P_{n-4}
\end{array}\right)
$$

Matrix formulation of $P_{n}$ and $Q_{n}$ can be given as

$$
\left(\begin{array}{c}
P_{n+4} \\
P_{n+3} \\
P_{n+2} \\
P_{n+1} \\
P_{n}
\end{array}\right)=\left(\begin{array}{ccccc}
1 & 1 & 1 & 1 & 1 \\
1 & 0 & 0 & 0 & 0 \\
0 & 1 & 0 & 0 & 0 \\
0 & 0 & 1 & 0 & 0 \\
0 & 0 & 0 & 1 & 0
\end{array}\right)^{n}\left(\begin{array}{c}
P_{4} \\
P_{3} \\
P_{2} \\
P_{1} \\
P_{0}
\end{array}\right)
$$

and

$$
\left(\begin{array}{c}
Q_{n+4} \\
Q_{n+3} \\
Q_{n+2} \\
Q_{n+1} \\
Q_{n}
\end{array}\right)=\left(\begin{array}{lllll}
1 & 1 & 1 & 1 & 1 \\
1 & 0 & 0 & 0 & 0 \\
0 & 1 & 0 & 0 & 0 \\
0 & 0 & 1 & 0 & 0 \\
0 & 0 & 0 & 1 & 0
\end{array}\right)^{n}\left(\begin{array}{c}
Q_{4} \\
Q_{3} \\
Q_{2} \\
Q_{1} \\
Q_{0}
\end{array}\right)
$$

Induction proofs may be used to establish the matrix formulations $P_{n}$ and $Q_{n}$. Similarly, matrix formulation of $V_{n}$ can be given as

$$
\left(\begin{array}{c}
V_{n+4} \\
V_{n+3} \\
V_{n+2} \\
V_{n+1} \\
V_{n}
\end{array}\right)=\left(\begin{array}{lllll}
1 & 1 & 1 & 1 & 1 \\
1 & 0 & 0 & 0 & 0 \\
0 & 1 & 0 & 0 & 0 \\
0 & 0 & 1 & 0 & 0 \\
0 & 0 & 0 & 1 & 0
\end{array}\right)^{n}\left(\begin{array}{c}
V_{4} \\
V_{3} \\
V_{2} \\
V_{1} \\
V_{0}
\end{array}\right) .
$$


Consider the matrices $N_{P}, E_{P}$ defined by as follows:

$$
\begin{aligned}
N_{P} & =\left(\begin{array}{ccccc}
4+2 i & 2+i & 1+i & 1 & 0 \\
2+i & 1+i & 1 & 0 & 0 \\
1+i & 1 & 0 & 0 & 0 \\
1 & 0 & 0 & 0 & i \\
0 & 0 & 0 & i & 1-i
\end{array}\right), \\
E_{P}= & \left(\begin{array}{cccccc}
G P_{n+4} & G P_{n+3} & G P_{n+2} & G P_{n+1} & G P_{n} \\
G P_{n+3} & G P_{n+2} & G P_{n+1} & G P_{n} & G P_{n-1} \\
G P_{n+2} & G P_{n+1} & G P_{n} & G P_{n-1} & G P_{n-2} \\
G P_{n+1} & G P_{n} & G P_{n-1} & G P_{n-2} & G P_{n-3} \\
G P_{n} & G P_{n-1} & G P_{n-2} & G P_{n-3} & G P_{n-4}
\end{array}\right) .
\end{aligned}
$$

Next Theorem presents the relations between $A^{n}, N_{P}$ and $E_{P}$.

Theorem 5.1. For $n \geq 4$, we have

$$
A^{n} N_{P}=E_{P}
$$

Proof. Using the relations

$$
\begin{aligned}
G P_{n} & =P_{n}+i P_{n-1}, \\
G P_{n+4} & =P_{n+4}+i P_{n+3}=(4+2 i) P_{n+1}+(4+2 i) P_{n}+(4+2 i) P_{n-1}+(3+2 i) P_{n-2}+(2+i) P_{n-3}, \\
G P_{n+3} & =(2+i) P_{n+1}+(2+i) P_{n}+(2+i) P_{n-1}+(2+i) P_{n-2}+(1+i) P_{n-3},
\end{aligned}
$$

we get $A^{n} N_{P}=E_{P}$.

Above Theorem can be proved by mathematical induction as well.

Consider the matrices $N_{Q}, E_{Q}$ defined by as follows:

$$
\begin{aligned}
N_{Q}= & \left(\begin{array}{ccccc}
15+7 i & 7+3 i & 3+i & 1+5 i & 5-i \\
7+3 i & 3+i & 1+5 i & 5-i & -1-i \\
3+i & 1+5 i & 5-i & -1-i & -1-i \\
1+5 i & 5-i & -1-i & -1-i & -1-i \\
5-i & -1-i & -1-i & -1-i & -1+9 i
\end{array}\right) \\
E_{Q}= & \left(\begin{array}{ccccc}
G Q_{n+4} & G Q_{n+3} & G Q_{n+2} & G Q_{n+1} & G Q_{n} \\
G Q_{n+3} & G Q_{n+2} & G Q_{n+1} & G Q_{n} & G Q_{n-1} \\
G Q_{n+2} & G Q_{n+1} & G Q_{n} & G Q_{n-1} & G Q_{n-2} \\
G Q_{n+1} & G Q_{n} & G Q_{n-1} & G Q_{n-2} & G Q_{n-3} \\
G Q_{n} & G Q_{n-1} & G Q_{n-2} & G Q_{n-3} & G Q_{n-4}
\end{array}\right) .
\end{aligned}
$$

The following Theorem presents the relations between $A^{n}, N_{Q}$ and $E_{Q}$. 
THEOREM 5.2. We have

$$
A^{n} N_{Q}=E_{Q}
$$

Proof. The proof requires some lengthy calculation, so we omit it.

The previous Theorem, also, can be proved by mathematical induction.

\section{Conclusions}

- In the section 1, we present some background about generalized Pentanacci numbers.

- In the section 2, we present Binet's formulas, generating functions, and the summation formulas for generalized Pentanacci numbers.

- In the section 3, first we recall Gaussian integers and then we define Gaussian generalized Pentanacci numbers and as special cases, we investigate Gaussian Pentanacci and Gaussian Pentanacci-Lucas numbers, with their properties such as the generating functions, Binet's formulas and sums formulas of these Gaussian numbers.

- In the section 4, we obtain some identities of Pentanacci numbers and Pentanacci-Lucas numbers and some identities of Gaussian Pentanacci numbers and Gaussian Pentanacci-Lucas numbers. Furthermore, we present Simson formulas of those numbers.

- In the section 5, we give some matrix formulation of generalized Pentanacci numbers and Gaussian generalized Pentanacci numbers.

\section{References}

[1] Asci M., Gurel E., Gaussian Jacobsthal and Gaussian Jacobsthal Polynomials, Notes on Number Theory and Discrete Mathematics, Vol.19, pp.25-36, 2013.

[2] Bacani, J. B., Rabago, J. F. T., On Generalized Fibonacci Numbers, Applied Mathematical Sciences, 9 (25), 3611-3622, 2015.

[3] Berzsenyi G., Gaussian Fibonacci Numbers, Fibonacci Quart., Vol.15(3), pp.233-236, 1977.

[4] Catarino, P., and Campos, H., A note on Gaussian Modified Pell numbers, Journal of Information \& Optimization Sciences, Vol. 39, No. 6, pp. 1363-1371, 2018.

[5] G. P. Dresden G. P., Du, Z., A Simplified Binet Formula for k-Generalized Fibonacci Numbers, J. Integer Seq. 17, art. 14.4.7, 9 pp., 2014.

[6] Fraleigh J.B., A First Course In Abstract Algebra, (2nd ed.), Addison-Wesley, Reading, ISBN 0-201-01984-1, 1976.

[7] E. Gurel, k-Order Gaussian Fibonacci and k-Order Gaussian Lucas Recurrence Relations, Ph.D Thesis, Pamukkale University Institute of Science Mathematics, Denizli, Turkey (2015).

[8] Halici S., Öz S., On some Gaussian Pell and Pell-Lucas numbers, Ordu University Science and Technology Journal, Vol.6(1), pp.8-18, 2016.

[9] Halici S., Öz S., On Gaussian Pell Polynomials and Their Some Properties, Palestine Journal of Mathematics, Vol 7(1), 251-256, 2018.

[10] Harman C. J., Complex Fibonacci Numbers, Fibonacci Quart., Vol.19(1), pp. 82-86, 1981.

[11] Horadam, A. F., Complex Fibonacci Numbers and Fibonacci quaternions, Amer. Math. Monthly 70, 289-291, 1963.

[12] Jordan J.H., Gaussian Fibonacci and Lucas Numbers, Fibonacci Quart., Vol.3, pp. 315-318, 1965. 
[13] Pethe S., Horadam A.F., Generalised Gaussian Fibonacci numbers, Bull. Austral. Math. Soc., Vol.33, pp.37-48, 1986.

[14] Melham, R. S., Some Analogs of the Identity $F_{n}^{2}+F_{n+1}^{2}=F_{2 n+1}^{2}$, Fibonacci Quarterly, 305-311, 1999.

[15] Natividad, L. R., On Solving Fibonacci-Like Sequences of Fourth, Fifth and Sixth Order, International Journal of Mathematics and Computing, 3 (2), 2013.

[16] Pethe S., Horadam A.F., Generalised Gaussian Lucas Primordial numbers, Fibonacci Quart., pp. 20-30, 1988.

[17] Pethe, S., Some Identities for Tribonacci Sequences, The Fibonacci Quarterly, 26, 144-151, 1988.

[18] Simson. R., An Explanation of an Obscure Passage in Albrecht Girard's Commentary upon Simon Stevin's Works, Philosophical Transactions of the Royal Society 48.1, 368-377, 1753.

[19] Rathore, G.P.S., Sikhwal, O., Choudhary, R., Formula for finding nth Term of Fibonacci-Like Sequence of Higher Order, International Journal of Mathematics And its Applications, 4 (2-D), 75-80, 2016.

[20] Sloane, N.J.A., The on-line encyclopedia of integer sequences, http://oeis.org/

[21] Soykan, Y., Taşdemir, E., Okumuş, İ., Göcen, M., Gaussian Generalized Tribonacci Numbers, Journal of Progressive Research in Mathematics(JPRM), 14 (2), 2373-2387, 2018.

[22] Soykan, Y., Gaussian Generalized Tetranacci Numbers, Journal of Advances in Mathematics and Computer Science, 31(3): 1-21, Article no.JAMCS.48063, 2019.

[23] Soykan, Y., Simson Identity of Generalized m-step Fibonacci Numbers, arXiv:1903.01313v1 [math.NT], 2019.

[24] Taşcı, D., and Acar, H., Gaussian Pentanacci Numbers, Communications in Mathematics ans Applications, 8 (3), 379-386, 2017.

[25] Taşc1, D., and Acar, H., Gaussian Padovan and Gaussian Pell-Padovan Numbers, Commun. Fac. Sci. Ank. Ser. A1 Math. Stat., Volume 67, Number 2, pp. 82-88, 2018.

[26] Yagmur, T., and Karaaslan, N., Gaussian Modified Pell Sequence and Gaussian Modified Pell Polynomial, Aksaray University Journal of Science and Engineering, Volume 2, Issue 1, pp. 63-72, 2018.

[27] Wolfram, D.A., Solving Generalized Fibonacci Recurrences, Fibonacci Quarterly, 129-145, 1998. 